\title{
Authors' reply: Should we inherit bursectomy or discard it?
}

\author{
Junya Fujita $\cdot$ Yukinori Kurokawa $\cdot$ \\ Shuji Takiguchi $\cdot$ Yuichiro Doki
}

Received: 26 October 2011/Accepted: 7 November 2011/Published online: 20 December 2011

(c) The International Gastric Cancer Association and The Japanese Gastric Cancer Association 2011

We appreciate the comments of Dr. Kayaalp and his colleagues regarding our article on the clinical trial evaluating bursectomy for gastric cancer patients [1]. We read their letter with interest and were rather surprised that surgeons in Turkey routinely perform bursectomy for radical gastrectomy, as, in Japan, this traditional technique has recently become obsolete. We agree with their theoretical considerations and we also had some doubts about the efficacy of prophylactic bursectomy, which was exactly the rationale for conducting our prospective randomized controlled trial (RCT).

The Japanese gastric cancer treatment guidelines provide an ambiguous recommendation without reliable evidence that bursectomy may be performed for tumors penetrating the serosa of the posterior gastric wall [2]. On the other hand, data analysis of the nationwide registry of gastric cancer in Japan revealed that $10.7 \%$ of patients with subserosal cancer developed peritoneal recurrence after resection [3], and gastric cancer patients diagnosed as having clinical T2 stage (muscularis propria or subserosa; Japanese classification of gastric carcinoma, 2nd English edition) had generally received prophylactic bursectomy in the standard D2 gastrectomy performed by Japanese surgeons in the 1990s. Taking this background into account, we designed our trial to include not only cT3-4 serosapositive patients but also cT2 serosa-negative patients in the eligibility criteria for our study. In fact, at the time of the interim analysis in this study, 23 patients had peritoneal

\footnotetext{
J. Fujita ( $\bowtie)$

Department of Surgery, Toyonaka Municipal Hospital,

Osaka, Japan

e-mail: jfujita@chp.toyonaka.osaka.jp

Y. Kurokawa $\cdot$ S. Takiguchi · Y. Doki

Department of Gastroenterological Surgery, Osaka University

Graduate School of Medicine, Osaka, Japan
}

recurrence, and 12 of these 23 patients had serosa-negative (pathological subserosal) tumors.

To reply to Dr. Kayaalp's question, the distribution of TNM stages in our entire patient cohort was comparable in the two groups (Stage I/II/III/IV; 44/30/27/3 in the bursectomy group and 57/22/22/5 in the non-bursectomy group), and the stage distribution was also comparable in pT3-4 patients (Stage II/III/IV; 6/18/1 in the bursectomy group and $6 / 13 / 3$ in the non-bursectomy group). As a result of the inaccuracy of macroscopic diagnosis of tumor depth during surgery, considerable numbers of pT1 patients were enrolled in this trial (16.3\% in the bursectomy group and $17.9 \%$ in the non-bursectomy group). As we mentioned in the article, the survival results for the pT3-4 patients were regarded as an under-powered subgroup analysis, and we were not convinced that the numbers of these patients were sufficient to lead to a definite conclusion. However, we regarded it as noteworthy that, in the entire cohort of patients, the nonbursectomy group showed worse overall survival, with a hazard ratio for death of 1.44, and the non-inferiority of the omission of bursectomy was denied in the analysis. Thus, we considered reexamining the effectiveness of bursectomy by focusing on a more promising cohort of patients. The Japan Clinical Oncology Group has launched a large-scale RCT (JCOG1001) targeting 1000 patients with cT3-T4 tumors (subserosa-serosa; TNM 7th edition) to assess the superiority of bursectomy, and this trial is now in progress with favorable recruitment of patients.

\section{References}

1. Fujita J, Kurokawa Y, Sugimoto T, Miyashiro I, Iijima S, Kimura Y, Takiguchi S, Fujiwara Y, Mori M, Doki Y. Survival benefit of 
bursectomy in patients with resectable gastric cancer: interim analysis results of a randomized controlled trial. Gastric Cancer. 2011. doi:10.1007/s10120-011-0058-9

2. Japanese Gastric Cancer Association. Japanese gastric cancer treatment guidelines 2010 (ver. 3). Gastric Cancer. 2011;14: $113-23$.
3. Japanese Gastric Cancer Association Registration Committee. Gastric cancer treated in 1991 in Japan: data analysis of nationwide registry. Gastric Cancer. 2006;9:51-66. 